\title{
Defect detection in stainless steel tubes with AMR and GMR sensors using remote field eddy current inspection
}

\author{
Dario J. L. Pasadas, A. Lopes Ribeiro, Helena G. Ramos, Tiago J. Rocha \\ Instituto de Telecomunicações and Instituto Superior Técnico/Universidade Técnica de Lisboa, Av. Rovisco Pais 1, 1049-001 Lisbon,Portugal
}

\section{ABSTRACT}

The purpose of this paper is to compare the performance of the giant magneto-resistor (GMR) and anisotropic magneto-resistor (AMR) sensors for remote field eddy current testing in stainless steel tubes. Two remote field eddy current probes were built to compare detection and characterization capabilities in standard defects like longitudinal and transverse defects. Both probes include a coil to produce a sinusoidal magnetic field that penetrates the tube wall. Each probe includes a detector with GMR and AMR sensors, where each sensor has four magneto-resistive elements configured in a Wheatstone bridge. Each sensor needs be biased differently to operate in the high sensitivity linear mode. The description of the measurement system used to detect defects is presented in this paper. For the choice of the detector's optimal position, numerical simulations and experimental measurements were performed. For comparison of these sensors in defect detection using remote field eddy current testing, experimental measurements were performed under the same conditions. The results are presented and discussed in this paper.

\section{Section: RESEARCH PAPER}

Keywords: eddy currents; remote field testing; tube inspection; AMR sensor; GMR Sensor

Citation: Dario J. L. Pasadas, A. Lopes Ribeiro, Helena G. Ramos, Tiago J. Rocha, Defect detection in stainless steel tubes with AMR and GMR sensors using remote field eddy current inspection, Acta IMEKO, vol. 4, no. 2, article 11, June 2015, identifier: IMEKO-ACTA-04 (5)-02 -11

Editor: Paolo Carbone, University of Perugia, Italy

Received November 26, 2014; In final form March 9, 2015; Published June 2015

Copyright: (C) 2015 IMEKO. This is an open-access article distributed under the terms of the Creative Commons Attribution 3.0 License, which permits unrestricted use, distribution, and reproduction in any medium, provided the original author and source are credited

Funding: Instituto de Telecomunicações project EvalTubes and projects: UID/EEA/50008/2013, SFRH/BD/81856/2011 and SFRH/BD/81857/2011 of the Portuguese Science and Technology Foundation (FCT)

Corresponding author: Dario J. L. Pasadas, e-mail: dpasadas@|x.it.pt

\section{INTRODUCTION}

The remote field eddy current testing (RFEC) is currently applied in non-destructive evaluation (NDE) of metallic tubes [1]-[3]. This testing method is a special case of eddy current testing (ECT) currently used to evaluate the metal thickness [4], [5] and to detect defects in the material [5], [6].

The RFEC testing is an electromagnetic technique that allows the inspection of discontinuities in the metallic materials under test. Remote field probes have an equal sensitivity to internal and external tube defects and the phase shift is directly proportional to wall metal loss. Detection, localization and characterization [7], [8] of defects are the three basic activities in the ECT field. The achievement of these three inspection actions continues to be in study by several researchers to improve the current systems used in industrial maintenance. The development of computers with good performance, simulation tools, advanced signal processing and new electromagnetic sensors are attracting many scientists to work in the NDE fields.
The remote field eddy current technique requires one coil excited with a time-varying current to produce a magnetic field that penetrates the tube wall under test. The excitation can be sinusoidal [9] or pulsed [10]. In this article the excitation current is sinusoidal with constant amplitude. The magnetic field produced by the excitation coil induces currents in the tube wall. These currents are called eddy currents. The field diffusion along the tube wall is the base of the through-wall indirect technique [11].

Analysing a material with a defect, the eddy current flow changes around the defect and the magnetic field produced by it also changes. This magnetic field perturbation is measured with a magnetic detector in order to evaluate the defect features.

Nowadays, small pick-up coils are the most used as magnetic detectors. Recently other magnetic detectors with improved characteristics were introduced, such as Hall effect sensors [12], [13] and magneto-resistor sensors like anisotropic magneto- 
Table 1. Magnetic sensors characteristics.

\begin{tabular}{lccc}
\hline Type of magnetic sensor & Sensitivity to & Magnetic Field range & Frequency range \\
\hline Coil & $d \Phi / d t$ & $\approx 1 \mathrm{nT}$ to more than $10 \mathrm{~T}$ & $\approx 3 \mathrm{kHz}$ to $\mathrm{more}$ than $5 \mathrm{MHz}$ \\
Hall & $B$ & $\approx 1 \mathrm{mT}$ to $10 \mathrm{~T}$ & $\mathrm{DC}$ to $10 \mathrm{kHz}$ \\
AMR & $H$ & $\approx 10 \mu \mathrm{T}$ to $1 \mathrm{~T}$ & $\mathrm{DC}$ to $1 \mathrm{MHz}$ \\
GMR & $H$ & $\approx 10 \mu \mathrm{T}$ to $10 \mathrm{~T}$ & $\mathrm{DC}$ to $1 \mathrm{MHz}$ \\
\hline
\end{tabular}

resistors (AMR) [14], [15] or giant magneto-resistors (GMR) [16], [17]. Table 1 summarizes the specifications of the magnetic sensors above mentioned.

In this paper, special attention has been given to the AMR and GMR sensors to provide advantages over coil based magnetic sensors. The directional characteristics, high sensitivity with linear response and large bandwidth (DC to 1 $\mathrm{MHz}$ ) provided by these sensors make them excellent candidates for defect detection applications in steel tubes.

\section{CHARACTERISTICS OF THE TUBE SAMPLE UNDER TEST}

The inspected material is a tube sample of austenitic stainless steel (AISI 304) with internal diameter of $26 \mathrm{~mm}$ and external diameter of $28 \mathrm{~mm}$. The magnetic permeability of this material is equal to $\mu_{0}=4 \pi \times 10^{-7} \mathrm{H} / \mathrm{m}$ and the electric conductivity is equal to $\sigma=1.4 \mathrm{MS} / \mathrm{m}$. This type of steel is non-ferromagnetic and is used in several industries due to its good resistance to corrosion. Some typical standard defects like longitudinal and transverse cracks were made into the tube sample in order to test the defect detection with AMR and GMR sensors. Figure 1 presents the two types of defects analysed in this paper.

\section{EXPERIMENTAL SETUP}

The experimental setup used in this work is illustrated in Figure 2. A single axis positioning system is used to move the probe along the inner tube wall with steps of $0.5 \mathrm{~mm}$. This positioning system is controlled through an RS232 interface by the personal computer. A data acquisition board (PXI-6251) included in a PXI System from National Instruments (NI), measures the output voltage (from GMR or AMR sensors), amplified by an instrumentation amplifier (INA118) with $40 \mathrm{~dB}$ of gain, and the voltage across the current sampling resistor $R s$ equal to $0.22 \Omega$. This resistor $R s$ is used to monitor the amplitude and phase of the excitation current. The data acquisition board has 16-bit of resolution and a maximum sampling rate of $1.25 \mathrm{MS} / \mathrm{s}$ per channel. The excitation coil current was sinusoidal and generated by a FLUKE5700A calibrator controlled through the GPIB Interface. The PXI

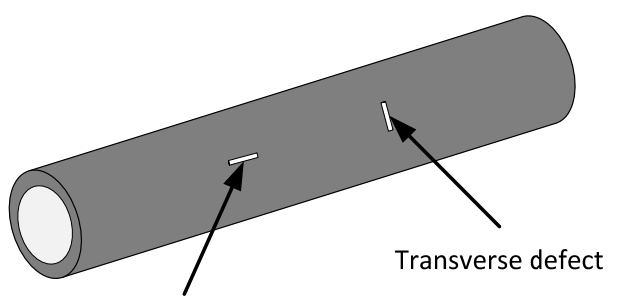

Longitudinal defect

Figure 2. Representation of the standard defects tested in this work.

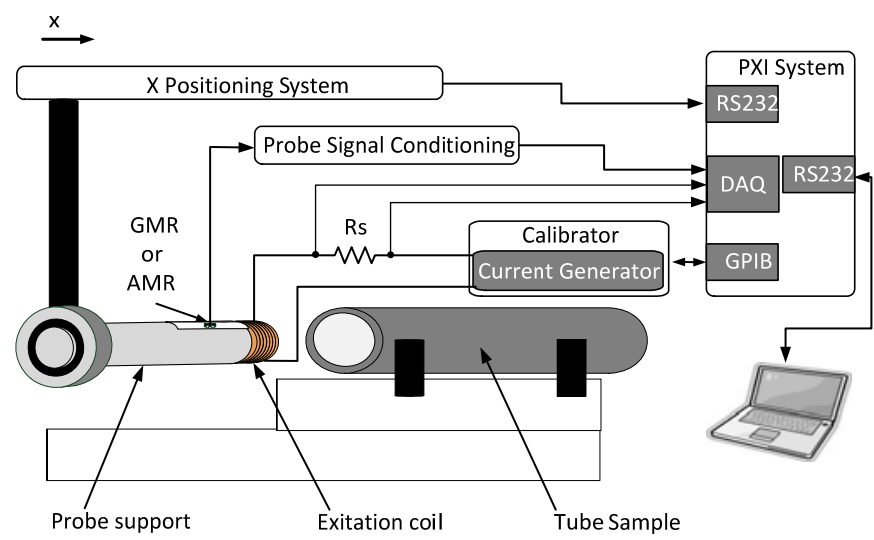

Figure 1. Representation of the experimental setup.

system was controlled through the RS232 interface by a personal computer running MATLAB program.

At each step of the probe movement, the sensor sinusoidal signal was acquired during 100 periods of the excitation current at the maximum sampling frequency of the board. For each measurement point, a three-parameter sine-fitting algorithm was used to extract the parameters of the sinusoidal sensor output and the excitation current. The amplitude of the sensor signal and the phase difference between the sensor signal and the excitation current are the parameters estimated in this work. The excitation frequency was $5 \mathrm{kHz}$. It takes $20 \mathrm{~ms}$ to acquire the signal at each point of the scan, but it is necessary to wait $0.5 \mathrm{~s}$ at each point to stabilize the probe.

\subsection{AMR Sensor}

The AMR sensor used in this work is the HMC 1021Z from Honeywell. This sensor has one single sensing axis with high sensitivity $(1 \mathrm{mV} / \mathrm{V} /$ gauss $)$ and a field range of \pm 6 gauss. The sensing axis is oriented along the longitudinal $\mathrm{x}$-axis of the tube. The HMC 1021Z sensor is configured in a Wheatstone bridge with four magneto-resistive elements. The power supply applied to the bridge was $12 \mathrm{~V}$. A set/reset drive circuit providing pulses of electrical current was used to force the sensor to operate in the high sensitivity and linear mode. This pulse of current was made before each data measurement.

\subsection{GMR Sensor}

The GMR sensor is the AA002-02 from Non Volatile Electronics. This sensor includes four magneto-resistive elements with GMR technology configured in a Wheatstone bridge, where two GMR elements are shielded, working as passive resistors and the other two are GMR sensing elements with resistance values changing linearly with the variation of the magnetic field. The bridge was powered by a power supply equal to $12 \mathrm{~V}$. Due to the output characteristic of the GMR sensor, a small permanent magnet was placed close to the sensor to polarise it in the high sensitivity and linear mode. 


\section{CHOICE OF THE OPTIMAL POSITION FOR THE DETECTOR}

The design of the probe for remote eddy current inspection is very important for detection optimization. First of all, it is necessary to determine the optimum distance between the magnetic sensor and the excitation coil. For this purpose, a finite element commercial program was used to study the remote field eddy current phenomenon along the inner wall of the stainless steel tube AISI 304. The simulation was made applying an excitation current to the coil with $150 \mathrm{~mA}$ at $5 \mathrm{kHz}$. The coil was placed in a fixed position into the tube as depicted in Figure 3. The magnetic field intensity along the inner tube wall was obtained and is presented in Figure 4.

The operating frequency was chosen considering the tube thickness and the relation to the standard depth of penetration. At this frequency the magnetic field diffuses easily to the outside of the stainless steel tube with thickness equal to $2 \mathrm{~mm}$.

When the RFEC technique is applied, three operating zones exist. Observing Figure 4 with the measured signal along the inner wall, the three operating zones are visible and are called direct zone $(0 \mathrm{~mm}<\mathrm{x}<0.3 \mathrm{~mm})$, transition zone $(0.3 \mathrm{~mm}<\mathrm{x}$ $<5 \mathrm{~mm})$ and remote zone $(\mathrm{x}>5 \mathrm{~mm})$. As the direct zone is close to the excitation coil, the magnetic field produced by the eddy currents is dominated by the strong influence of the magnetic field produced by the excitation coil which doesn't allow the detection of the defect. The remote zone is the region where the magnetic field is dominated by the field produced by the eddy currents and the field intensity decreases exponentially. When a defect is present in the tube wall, the perturbation of the magnetic field produced by the deviation of the eddy currents from the defect can be sensed by a magnetic sensor placed in that zone. A transition zone is visible in Figure 4 that

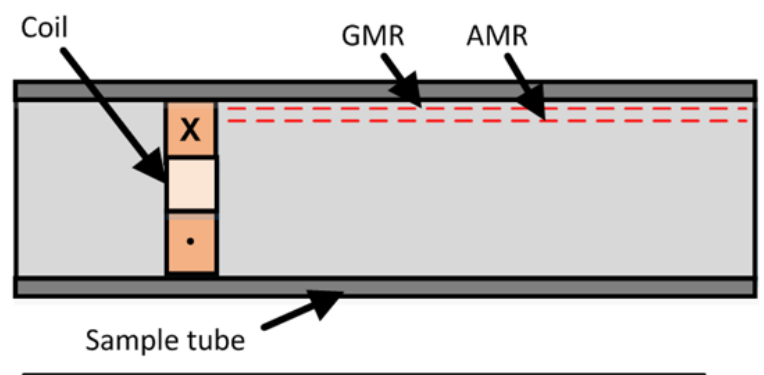

- Magnetic field paths along the inner wall tube.

Figure 3. Illustration of the experimental geometry including the sensor's path to obtain simulation results.

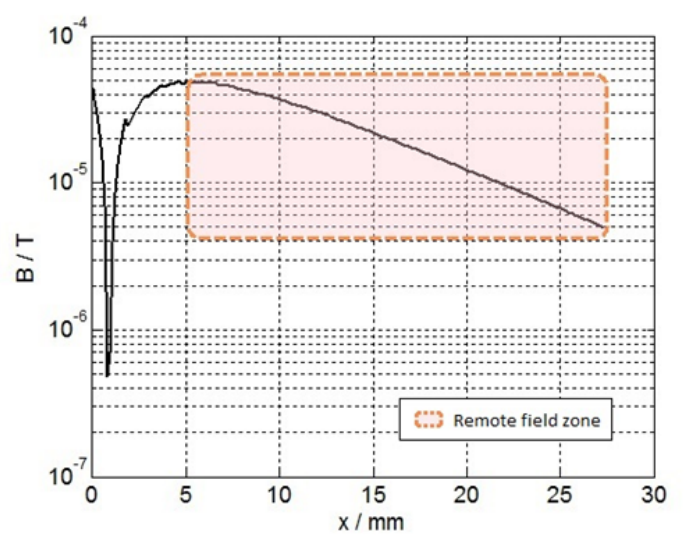

Figure 4. Simulated magnetic field obtained with the finite element model. corresponds to the region where the magnetic field produced by the eddy currents begins to overlap the excitation magnetic field and creates a minimum $(\mathrm{x}=1 \mathrm{~mm})$ in the total magnetic field amplitude due to their opposing phases.

Experimental results were obtained fixing the excitation coil into the tube and moving the AMR and GMR sensors along the inner wall in order to experimentally visualize the RFEC phenomena in a stainless steel AISI 304, validate the model, and to choose the optimal position of the sensor. Figure 5 depicts the magnetic field intensity along the inner tube wall, obtained experimentally with the AMR and GMR sensors. The direct and transition zones are not visible due to the size of the package of the sensor that does not allow measuring the magnetic field close to the excitation coil.

However, it is possible to observe the final part of the transition zone that corresponds to the maximum amplitude obtained in Figure 5. The information given in Figure 5 shows that the distance between the excitation coil and the detector must be greater than $3 \mathrm{~mm}$ to ensure that the measured field is inside the remote zone. The magnetic field difference between the AMR and GMR output sensors may originate from one factor. This difference is due to the different distances between the detectors and the tube wall. The GMR paths are closer to the tube wall due to its smaller size.

\section{EXPERIMENTAL RESULTS IN DEFECT DETECTION}

Two probes were mounted and tested for defect detection in the stainless steel tubes. In one probe the magnetic sensor is used to detect longitudinal defects in the tube wall and the other one transverse effects. Figure 6 shows photographs of both probes. To ensure that the detectors are in the remote zone, the distance between the excitation coil and the detectors was chosen $15 \mathrm{~mm}$.

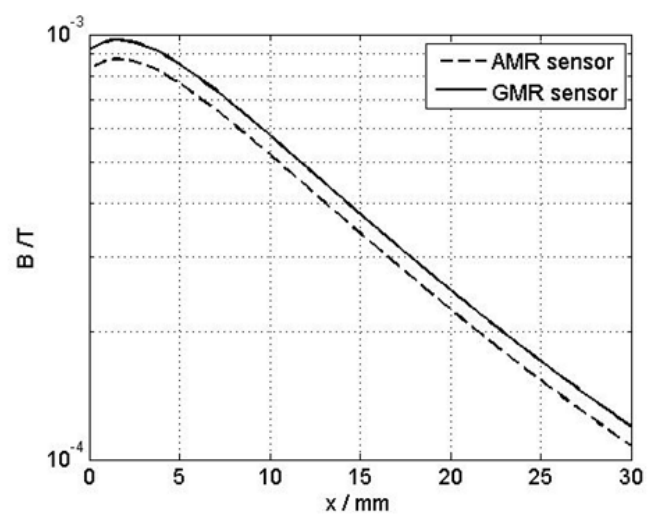

Figure 5. Magnetic field line along the tube wall obtained with the experimental setup.

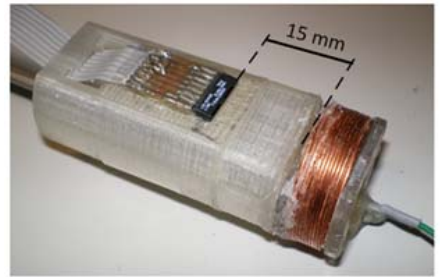

(a)

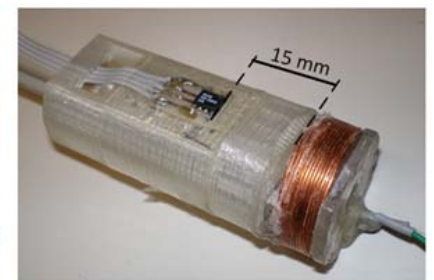

(b)
Figure 6. Photographs of the two mounted probes: (a) AMR probe with 10212 sensor from HMC; (b) GMR probe with AA002-02 sensor from NVE. 
For testing purposes a longitudinal defect and a transverse defect both $10 \mathrm{~mm}$ in length, were scanned using the experimental setup with each probe.

The first experimental test was made moving the AMR probe along the inner tube wall in the sample that has a longitudinal defect. A sinusoidal current with amplitude equal to $150 \mathrm{~mA}$ was applied to the excitation coil. The second test was scanning the same defect with the GMR probe. The output amplitudes of both sensors are depicted in Figure 7. The phase difference between the excitation current and each detector signal was also measured and depicted in Figure 8. It should be noted that the sensing axis of both sensors were directed along the longitudinal direction, close to the inner tube wall.

Observing Figures 7 and 8, an amplitude perturbation and phase perturbation is visible when the probes passed close the longitudinal defect. The perturbation occurred between the positions $\mathrm{x}=45 \mathrm{~mm}$ and $\mathrm{x}=55 \mathrm{~mm}$ and corresponds to the defect position when the detector passed the defect. The defect zone is denoted by two orange lines.

Observing the output amplitudes of the sensors depicted in Figure 7, a perturbation is present but it is not possible to conclude anything about the geometry of the defect. Also, the amplitude signals contain noise due to lift-off effect (distance between sensor and the tube wall).

Observing the phase perturbation depicted in Figure 8, the defect zone is clearly identified by a negative phase peak, matching approximately the real length of the longitudinal defect. The unexpected perturbation represented in Figure 7 and Figure 8 between $x=20 \mathrm{~mm}$ and $\mathrm{x}=40 \mathrm{~mm}$ is caused by the

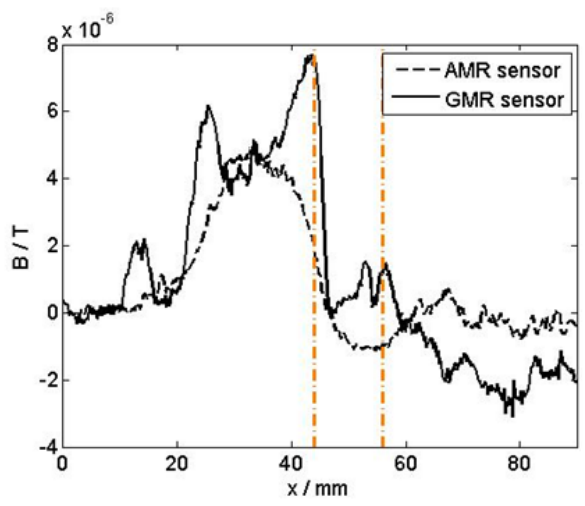

Figure 7. Output amplitudes (in tesla units) obtained with the AMR and GMR sensors for detection of a longitudinal defect, applying a sinusoidal current with amplitude $150 \mathrm{~mA}$ to the excitation coil.

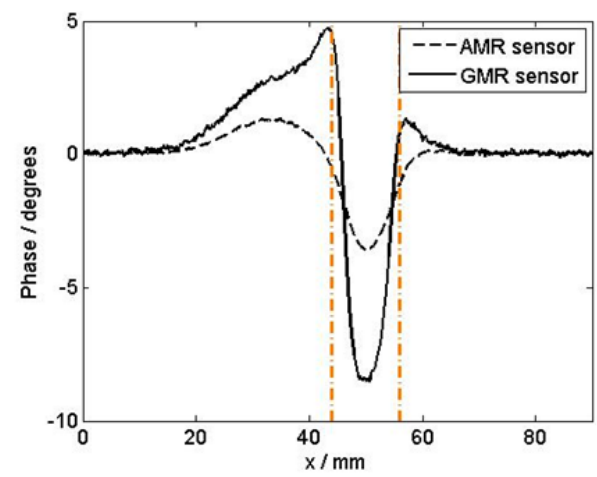

Figure 8. Phase difference between the excitation current and each sensor signal for defect detection of a longitudinal defect applying a sinusoidal current with amplitude $150 \mathrm{~mA}$ to the excitation coil. passage of the excitation coil under the defect, changing the eddy current amplitudes.

The same experimental test was made for both probes applying a sinusoidal current with amplitude $50 \mathrm{~mA}$ to the excitation coil. Figures 9 and 10 show the amplitude perturbation and phase perturbation when the probes passed close the defect.

In Figure 9 the attenuation of the amplitude perturbation in both output signals is visible. However, in Figure 10 it is seen that the phase perturbation continues to be present between $\mathrm{x}=45 \mathrm{~mm}$ and $\mathrm{x}=55 \mathrm{~mm}$ with approximately the same variation type shown in Figure 8 for different amplitudes. This shows that the phase difference between the excitation current and the detector signal can be useful to detect and characterize defects with approximately the same information, but different excitation current amplitudes.

The same comparison was made analysing the transverse defect. The results for the phase perturbation applying a sinusoidal amplitude current of $150 \mathrm{~mA}$ and $50 \mathrm{~mA}$ are depicted in Figure 11 and Figure 12, respectively. The defect position is marked with an orange line.

Figures 11 and 12 show that the defect position is clearly identified by a negative peak for each probe. The unexpected perturbation presented when the longitudinal defect was scanned is not present when the excitations coil crosses the defect position. This is due to the circumferential nature of the excitation current, the eddy current direction being parallel to the defect and the sensing axis of the detectors.

It is also visible that the phase perturbation obtained

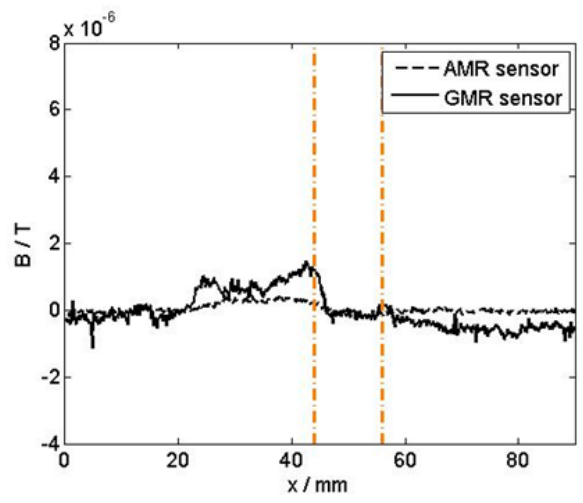

Figure 9. Output amplitudes (in tesla units) obtained with the AMR and GMR sensors for detection of a longitudinal defect, applying a sinusoidal current with amplitude $50 \mathrm{~mA}$ to the excitation coil.

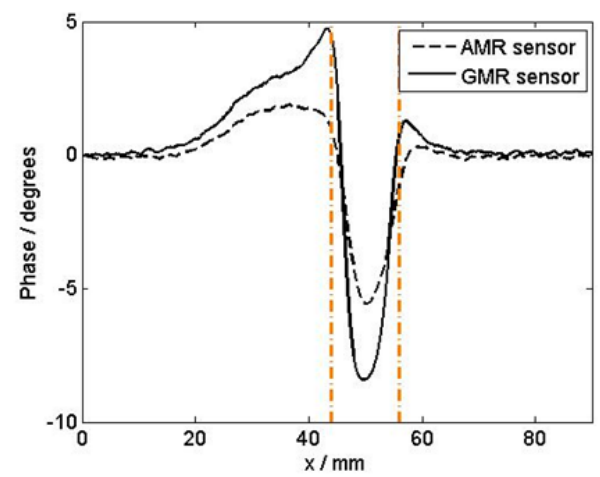

Figure 10. Phase difference between the excitation current and each sensor signal for defect detection of a longitudinal defect applying a sinusoidal current with amplitude $50 \mathrm{~mA}$ to the excitation coil. 


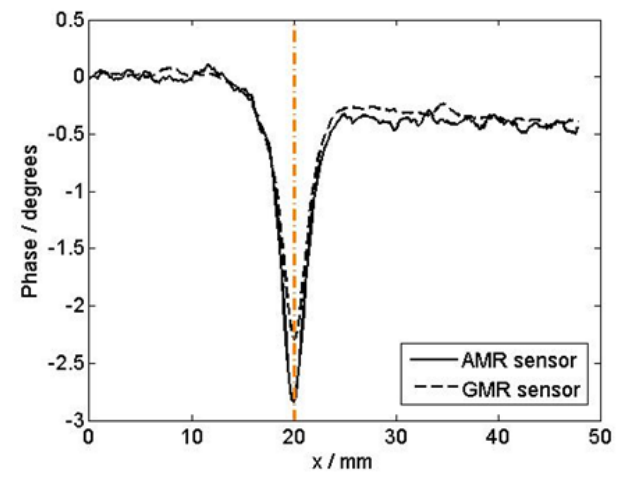

Figure 7. Phase difference between the excitation current and each sensor signal for defect detection of a transversal defect applying sinusoidal current with amplitude $150 \mathrm{~mA}$ to the excitation coil.

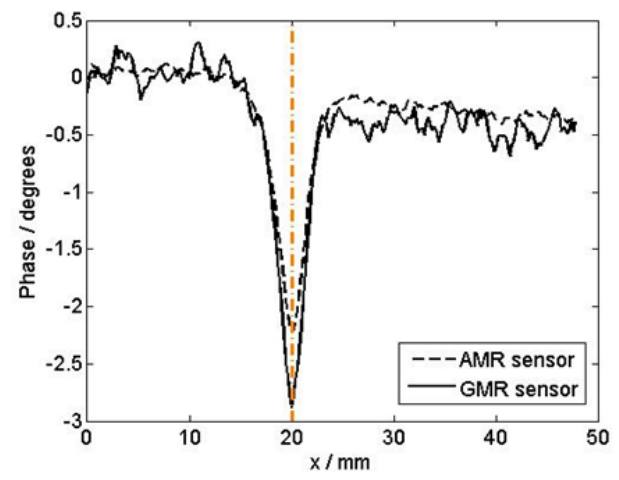

Figure 8. Phase difference between the excitation current and each sensor signal for defect detection of a transversal defect applying a sinusoidal current with amplitude $50 \mathrm{~mA}$ to the excitation coil.

between the excitation current and each sensor signal is maintained when a sinusoidal amplitude current of $150 \mathrm{~mA}$ or $50 \mathrm{~mA}$ is applied to the excitation coil.

\section{CONCLUSIONS}

The probes presented in this paper proved that AMR and GMR sensors as detectors seem to be good alternatives to conventional remote field eddy current probes that use coils as detectors. The main difference between these magneto-resistive sensors and coils is the possibility to use low frequency tests that allow an easy magnetic field penetration into thick wall tubes. When coils are used it is usually necessary to increase the frequency of operation to obtain good signal to noise ratios.

Experimental results proved that these sensors are able to detect defects in stainless steel tubes using remote field eddy current testing. The directional characteristics and high sensitivity with linear response over a large bandwidth (DC to 5 $\mathrm{MHz}$ ) provided by these sensors can be useful to detect subsurface defects at lower frequencies, where coil detectors can't be used.

The results clearly show that the GMR sensor is more sensitive to the magnetic field than the AMR sensor. The results also show that the phase perturbation contains more clear information about defect presence when compared to the amplitude perturbation. Furthermore, the phase perturbation remained unchanged for both 150 and $50 \mathrm{~mA}$ of excitation current, while the amplitude perturbation decreased with a decrement of the excitation current. This means that when the excitation current amplitude decreases, the amplitude output signal of both sensors becomes difficult to measure, however the phase still contains useful information caused by the defect presence.

Note that the chosen distance between the excitation coil and the detector is important to ensure that the measured field perturbation of the eddy currents is strong enough to detect defects with minimal signal attenuation.

As future work we consider testing these sensors on stainless steel tubes with larger thicknesses and using lower frequencies in order to increase the penetration depth of eddy currents, and to apply this method to other industrial applications.

Another important conclusion is related to the accuracy of the measurement of the geometrical sizes of the defects. Taken into consideration the phase measurements it is possible to conclude that we always got uncertainties under $5 \mathrm{~mm}$ for longitudinal defects. This quantity refers to the positions of the defect edges. For transverse circular defects the uncertainty is much lower, especially if the defect is perfectly circularly oriented.

\section{ACKNOWLEDGEMENT}

This work was developed under the Instituto de Telecomunicações project EvalTubes and supported by the projects: UID/EEA/50008/2013, SFRH/BD/81856/2011 and SFRH/BD/81857/2011 of the Portuguese Science and Technology Foundation (FCT). This support is gratefully acknowledged.

\section{REFERENCES}

[1] Y-J Kim, S-S Lee, "Eddy current probes of inclined coils for increased detectability of circumferential cracks in tubing", NDT \& E International, July, 2012, Vol. 49, pp. 77-82.

[2] D. H. Hur, M. S. Choi, D. H. Lee, S. J. Kim, J. H. Han, “A case study on detection and sizing of defects in steam generator tubes using eddy current testing", Nuclear Engineering and Design, January, 2010, Vol. 240, No. 1, pp. 204-208.

[3] J.Bruce Nestleroth, Richard J. Davis, "Application of eddy currents induced by permanent magnets for pipeline inspection", NDT \& E International, January, 2007, Vo. 40, No. 1, pp. 77-84.

[4] Helena G. Ramos, Tiago Rocha, Jakub Král, Dario Pasadas, Artur L. Ribeiro, "An SVM approach with electromagnetic methods to assess metal plate thickness", Measurement, August, 2014, Vol. 54, pp. 201-206.

[5] Tianlu Chen, Gui Yun Tian, Ali Sophian, Pei Wen Que, "Feature extraction and selection for defect classification of pulsed eddy current NDT", NDT \& E International, September, 2008, Vol. 41, No. 6, pp. 467-476.

[6] D. Pasadas, T. Rocha, H. G. Ramos, A. L. Ribeiro, "Evaluation of portable ECT instruments with positioning capability", Measurement, January, 2012, Vol. 45, No. 1, pp. 393-404.

[7] D. Kim, L. Udpa, S. Udpa, "Remote field eddy current testing for detection of stress corrosion cracks in gas transmission pipelines", Materials Letters, June, 2004, Vol. 58, No. 15, pp. 2101-2104.

[8] Dario Pasadas, Tiago Rocha, Artur L. Ribeiro, Helena G. Ramos, "ECT Characterization of a Linear Defect from Multiple angle Measurements", 19th Symposium IMEKO TC 4 Symposium and 17th IWADC Workshop Advances in Instrumentation and Sensors Interoperability, Barcelona, Spain, July 18-19, 2013, pp. 218-221.

[9] A. L. Ribeiro, H. G. Ramos, J. Gonçalves; "Thickness Measurement of a Nonmagnetic Metallic Plate Using Harmonic Eddy Current Excitation and a GMR Sensor", Proc IMEKO TC4 Symp., Natal, Brazil, , September, 2011, pp. 1-6. 
[10] B. Yang, X. li, "Pulsed remote field technique used for nondestructive inspection of ferromagnetic tube", NDT\&E International, January, 2013, Vol. 53, pp. 47-52.

[11] D. L. Atherton, S. Sullivan, "The Remote-Field Through-Wall Electromagnetic Inspection Technique for Pressure Tubes", Materials Evaluation, December, 1986, vol. 44, No. 13, pp. 1544-1550.

[12] Y. He, F. Luo, M. Pan, F. Weng, X. Hu, J. Gao, B. Lui, "Pulse Eddy Current Technique for Defect Detection in Aircraft Riveted Structures", NDT\&E International, , Mar. , 2010, Vol. 43, No. 2, pp. 176-181.

[13] K. Kosmas, C. Sargentis, D. Tsamakis, E. Hristoforou, "Nondestructive evaluation of magnetic metallic materials using hall sensors", Sensors and Actuators A: Physical, 2005, Vol. 161, No 1-2, pp. 359-362.

[14] D.J. Pasadas, A. Lopes Ribeiro, T. J. Rocha, H. G. Ramos, "Remote Field Eddy Current NDT in Tubes Using Anisotropic
Magneto-Resistors", Proc. 13th IMEKO TC10 Workshop on Technical Diagnostics Advanced measurement tools in technical diagnostics for systems reliability and safety, Warsaw, Poland, June 26-27, 2014, pp. 60-64.

[15] A. Jander, C. Smith, and R. Shneider, "Multi-frequency ect with amr sensor", NDT \& E International, September, 2011, Vol. 44, No. 5, pp. 438-441.

[16] L. Kufrin, O. Postolache, A. L. Ribeiro, H. G. Ramos, "Image Analysis for Crack Detection", Proc IEEE International Instrumentation and Technology Conf., Austin, United States, May, 2010, Vol. 1, pp. 1096 - 1100.

[17] J.H. Espina-Hernández, E. Ramírez-Pacheco, F. Caleyo, J. A. Pérez-Benitez, J.M. Hallen, "Rapid estimation of artificial nearside crack dimensions in aluminium using a GMR-based eddy current sensor", NDT \& E International, October, 2012, Vol. 51, pp. $94-100$. 